
in Mathematics in Computer Science, Birkhäuser Mathematics, Springer, ISSN 1661-8270 (print), ISSN 1661-8289 (electronic).

\title{
Dynamics and optimal control of Ebola transmission
}

\author{
Amira Rachah · Delfim F. M. Torres
}

Submitted 16-July-2015 / Accepted, after a revision, 10-Mar-2016.

\begin{abstract}
A major Ebola outbreak occurs in West Africa since March 2014, being the deadliest epidemic in history. As an infectious disease epidemiology, Ebola is the most lethal and is moving faster than in previous outbreaks. On 8 August 2014, the World Health Organization (WHO) declared the outbreak a public health emergency of international concern. Last update on 7 July 2015 by WHO reports 27609 cases of Ebola with a total of 11261 deaths. In this work, we present a mathematical description of the spread of Ebola virus based on the SEIR (Susceptible-Exposed-Infective-Recovered) model and optimal strategies for Ebola control. In order to control the propagation of the virus and to predict the impact of vaccine programmes, we investigate several strategies of optimal control of the spread of Ebola: control infection by vaccination of susceptible; minimize exposed and infected; reduce Ebola infection by vaccination and education.
\end{abstract}

Keywords Mathematical modelling · optimal control · epidemiology · Ebola virus · 2014 Ebola epidemic in West Africa.

\section{Introduction}

The most widespread epidemic of Ebola virus disease in history is currently ongoing in West Africa. Ebola virus is the most complex and lethal pathogen

Amira Rachah

Mathématiques pour l'Industrie et la Physique,

Institut de Mathématiques de Toulouse, Université Paul Sabatier,

F-31062 Toulouse Cedex 9, France

E-mail: arachah@math.univ-toulouse.fr

Delfim F. M. Torres (corresponding author)

Center for Research and Development in Mathematics and Applications (CIDMA),

Department of Mathematics, University of Aveiro, 3810-193 Aveiro, Portugal

E-mail: delfim@ua.pt 
for humans [7,19]. The first outbreak was in 1976 in Congo, close to Ebola river, where the disease takes its name. The Ebola virus causes an acute, often fatal haemorrhagic illness. The current outbreak in West Africa is the largest and most complex Ebola outbreak since the virus discovery. The increasing of this virulent virus is extremely rapid and has evolved into a health and humanitarian catastrophe of historic scope. The incubation period, that is, the time interval from infection with the virus to the onset of symptoms, is 2 to 21 days. Humans are not infectious to another person until they develop symptoms. Symptoms typically begin with sudden onset of fever, sore throat, muscle pain, and headaches. Then, vomiting, diarrhoea and rash usually follow, along with decreased function of the liver and kidneys. At this time infectious individuals begin to bleed, both internally and externally (bleeding from nose, mouth, eyes and anus). In final stage, these symptoms are followed by death 10, $13,18,20,24,31,33$. Ebola spreads through human-to-human transmission via close and direct physical contact (through broken skin or mucous membranes) with infected bodily fluids. The most infectious fluids are blood, faeces and vomit secretions; however, all body fluids have the capacity to transmit the virus. The virus is also transmitted indirectly via exposure to objects or environment contaminated with infected secretions. Because of this, healthcare workers must practice strict infection prevention and control precautions.

In epidemiology, mathematical models are a key tool that contribute to the understanding of the dynamics of a virus and the impact of vaccination programmes. More precisely, Mathematics has an important role in the study of propagation of virus spreads by allowing policy-makers to predict the impact of particular vaccine programmes or to derive more efficient strategies based on mathematical insights 26 29. In recent years, optimal control theory has become a powerful mathematical tool to assess the intervention of public health authorities. Indeed, the inclusion, in an epidemic model, of some practical control strategies, like vaccines, social distancing or quarantine, provides a rational basis for policies designed to control the spread of the virus. In this spirit, the aim of this work is to investigate effective strategies to control the spread of the Ebola virus by setting appropriate optimal control problems subject to a SEIR epidemic model that divides the population into four groups: the Susceptible, the Exposed (infected but not infectious), the Infectious, and the Recovered 1, 5, 11, 12,35. For a comparison study between the SIR and SEIR models we refer the reader to 23 . The main practical strategy of optimal control adopted is a vaccine $6,8,14,16,30$. For fractional (non-integer order) models see [2,3].

The paper is organized as follows. In Section 2 we present the mathematical model that describes the dynamics of the spread of Ebola virus in West Africa. In Section 3 we study several control strategies for the propagation of the virus by using the proposed model. In these strategies, we use parameters estimated from recent statistical data based on the WHO report of the 2014 Ebola outbreak 34. In Sections 3.1 and 3.2 we study the case of control through vaccination treatment by considering two different objective functions. In Section 3.3 we consider educational campaigns as a control, coupled with the 


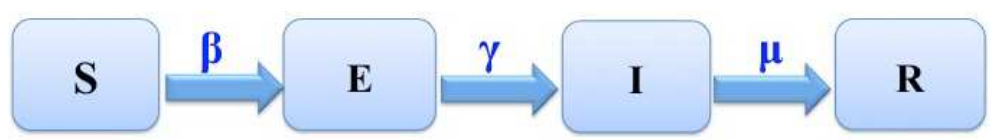

Fig. 1 Compartment diagram of the Susceptible-Exposed-Infectious-Recovered (SEIR) model (2).

vaccination treatment. We end with Section 4 of discussion of the numerical results and with Section [5 of conclusions and future work.

\section{The basic model equations}

In this section, we describe the transmission of Ebola virus by a SEIR model, where the population is divided into four groups: the susceptible individuals at time $t$, denoted by $S(t)$, enter the exposed class $E(t)$ before they become infectious. The infectious class at time $t$, denoted by $I(t)$, represents the individuals that are infected with the disease and are suffering the symptoms of Ebola. Finally, we have the recovered class, which at time $t$ is denoted by $R(t)$. The total population, assumed constant during the short period of time under study, is given by

$$
N=S(t)+E(t)+I(t)+R(t)
$$

at any instant of time $t$. The transmission of Ebola virus is then described by the following set of nonlinear ordinary differential equations (ODEs):

$$
\left\{\begin{array}{l}
\frac{d S(t)}{d t}=-\beta S(t) I(t), \\
\frac{d E(t)}{d t}=\beta S(t) I(t)-\gamma E(t), \\
\frac{d I(t)}{d t}=\gamma E(t)-\mu I(t), \\
\frac{d R(t)}{d t}=\mu I(t) .
\end{array}\right.
$$

Transitions between different states are described by the following parameters:

- the transmission rate $\beta$,

- the infectious rate $\gamma$,

- the recovered rate $\mu$.

Figure 1 shows the relationship between the variables of model (2). Its numerical resolution is studied by Rachah and Torres in 22. See also 21 for a simpler SIR model describing Ebola.

Remark 1 The model we consider here is very simple and assumes that the population is constant in the period of time under study: the sum of the righthand side of the equations of system (2) is zero. The possibility to design more 
complex models for a non-constant population is possible: see the recent eight state-variables model of [3]. However, for the analysis and simulation of the model to be possible, the authors of [3] imposed conditions to maintain the population $N$ constant. As remarked at the end of [3], the design of strategies when the population is not constant seems to be a good topic of research.

\section{Strategies for the control of the virus}

Recently, epidemiological models have used optimal control techniques. Most works focus on HIV, tuberculosis (TB) and dengue [8, 14, 25, 26, 28, 29. Control efforts are carried out to limit the spread of the disease and, in some cases, to prevent the emergence of drug resistance. In this section, we formulate several optimal control problems subject to the SEIR model (2), in order to derive the optimal treatment strategies. For each strategy, we study a specific objective in order to minimize not only the number of infected individuals and the systemic costs of treatments, but also to include an educational campaign with the vaccination treatment. The integration of educational campaigns has a great importance in countries that do not have the capacity to defend themselves against the virus. We compare the result of each strategy with the simulation results previously studied by Rachah and Torres in [22, the so called Strategy 1, which is described in Section 3.1. Strategies 2 and 3 are an improvement of the Strategy 1 of [22, and are given in Sections 3.2 and 3.3. respectively. More precisely, Strategy 3 consists in the study of an educational campaign (about the virus) coupled with a vaccination treatment. A comparison between the different strategies and the simulation results is given in Section 4.

Two approaches are common in optimal control: direct and indirect methods 27. The direct methods are based in the discretization of the optimal control problems, reducing them to nonlinear constrained optimization problems. Indirect methods are based on the Hamiltonian and the Pontryagin maximum principle. In this work we have adopted the direct approach. We have computed the numerical solutions of the optimal control problems with the help of the ACADO solver [4. ACADO is based on a multiple shooting method, including automatic differentiation and based ultimately on the semidirect multiple shooting algorithm of Bock and Pitt [9. The ACADO solver comes as a selfcontained public domain software environment, written in $\mathrm{C}++$, for automatic control and dynamic optimization. The reader interested in the details of the implemented algorithms is referred to [15].

\subsection{Strategy 1}

In this subsection, we present the optimal control problem investigated in [22], obtained by introducing into the model (2) a control $u(t)$ representing the vaccination rate at time $t$. The control $u(t)$ is the fraction of susceptible individuals being vaccinated per unit of time. Then, the mathematical model 
with control is given by the following system of nonlinear differential equations:

$$
\left\{\begin{array}{l}
\frac{d S(t)}{d t}=-\beta S(t) I(t)-u(t) S(t), \\
\frac{d E(t)}{d t}=\beta S(t) I(t)-\gamma E(t), \\
\frac{d I(t)}{d t}=\gamma E(t)-\mu I(t), \\
\frac{d R(t)}{d t}=\mu I(t)+u(t) S(t) .
\end{array}\right.
$$

The goal of the strategy is to reduce the infected individuals and the cost of vaccination. Precisely, the optimal control problem consists of minimizing the objective functional

$$
J(u)=\int_{0}^{t_{\text {end }}}\left[I(t)+\frac{\tau}{2} u^{2}(t)\right] d t,
$$

where $u(t)$ is the control variable, which represents the vaccination rate at time $t$, and the positive parameters $\tau$ and $t_{\text {end }}$ denote, respectively, the weight on cost and the duration of the vaccination program.

\subsection{Strategy 2}

We now improve the strategy of Section 3.1 by studying other strategy in order to better control the propagation of the spread of Ebola into populations. Our goal in this strategy is to reduce the number of exposed and infected individuals. More precisely, our optimal control problem consists of minimizing the objective functional

$$
J(u)=\int_{0}^{t_{\text {end }}}\left[A_{1} E(t)+A_{2} I(t)+\frac{\nu}{2} u^{2}(t)\right] d t
$$

subject to the model described by (3). The two first terms in the functional objective (5) represent benefit of $E(t)$ and $I(t)$ populations that we wish to reduce; $A_{1}$ and $A_{2}$ are positive constants to keep a balance in the size of $E(t)$ and $I(t)$, respectively. In the quadratic term of (5), $\nu$ is a positive weight parameter associated with the control $u(t)$, and the square of the control variable reflects the severity of the side effects of the vaccination. One has $u \in \mathcal{U}_{a d}$, where

$$
\mathcal{U}_{\text {ad }}=\left\{u: u \text { is measurable, } 0 \leq u(t) \leq u_{\max }<\infty, t \in\left[0, t_{\text {end }}\right]\right\}
$$

is the admissible control set, with $u_{\max }=0.9$. Note that it is not realistic to admit the possibility to vaccinate everybody and the value 0.9 means that, at maximum, $90 \%$ of susceptible are vaccinated. In the numerical simulations of model (2) and Strategy 1 of optimal control, Rachah and Torres [22] used 
the parameters estimated on November 2014 by Althaus and Kaurov [1,17, who studied statistically the data of the World Health Organisation (WHO) 34. In particular, in the statistical study [17, Kaurov studies the outbreak by modeling it with Wolfram's Mathematica language.

In order to compare our improvement of the optimal control study with the previous results of 22 , we use here the same parameters, that is, the same rate of infection $\beta=0.2$, the infectious rate $\gamma=0.1887$, the same recovered rate $\mu=$ 0.1 , and the same initial values $(S(0), E(0), I(0), R(0))=(0.88,0.07,0.05,0)$ for the initial number of susceptible, exposed, infected, and recovered populations (at the beginning, $88 \%$ of population is susceptible, $7 \%$ is exposed and $5 \%$ is infected with Ebola). Figures 2, 3, 4 and 5) show, respectively, the significant difference in the number of susceptible, recovered, exposed and infected individuals with Strategy 1, Strategy 2, and without control. In Figure 2, we see that the number of susceptible $S$, in case of optimal control under Strategy 2, decreases more rapidly during the vaccination campaign. It reaches $2 \%$ at the end of the campaign, in contrast with the $6.6 \%$ at the end of the campaign with Strategy 1, and against $17.3 \%$ in the absence of optimal control. Figure 3 shows that the number of recovered individuals increases rapidly. The number $R\left(t_{\text {end }}\right)$ at the end of the optimal control vaccination period of Strategy 2 is $97.9 \%$, instead of $93.3 \%$ in case of Strategy 1 , and against $81.2 \%$ without control. Figure 4 presents the time-dependent curve of exposed individuals. This curve shows that there is no peak of the curve of exposed individuals in case of control with Strategy 1 and Strategy 2 when we compare it with the curve in case without control, which shows a high peak. When we compare Strategy 1 and Strategy 2, we see that the curve of exposed individuals decreases more rapidly in case of control with Strategy 2. The period of incubation of the virus is only 30 days in case of control with Strategy 2 instead of 50 days in Strategy 1. In Figure 5, the time-dependent curve of infected individuals shows that the peak of the curve of infected individuals is less important in case of control with Strategy 2. In fact, the maximum value on the infected curve $I$ under optimal control is $7 \%$ in case of Strategy 2, instead of $7.56 \%$ in Strategy 1, and against 14\% without any control (see Figure 5). The other important effect of Strategy 2, which we see in the same curve, is the period of infection, which is the less important. The value of the period of infection is 65 days in case of Strategy 2, instead of 80 days in case of Strategy 1, and against more than 100 days without vaccination. This shows the efficiency of vaccination with Strategy 2 in controlling Ebola. Figure 6 gives a representation of the optimal control $u(t)$ for Strategy 1 and Strategy 2. The minimum values of the $J(u)$ functionals (4) and (5) are, respectively, 1,936 (Strategy 1) and 2,509 (Strategy 2).

\subsection{Strategy 3}

In this strategy we use the fact that individuals can acquire immunity against the virus either through educational campaigns or recovery after treatment 


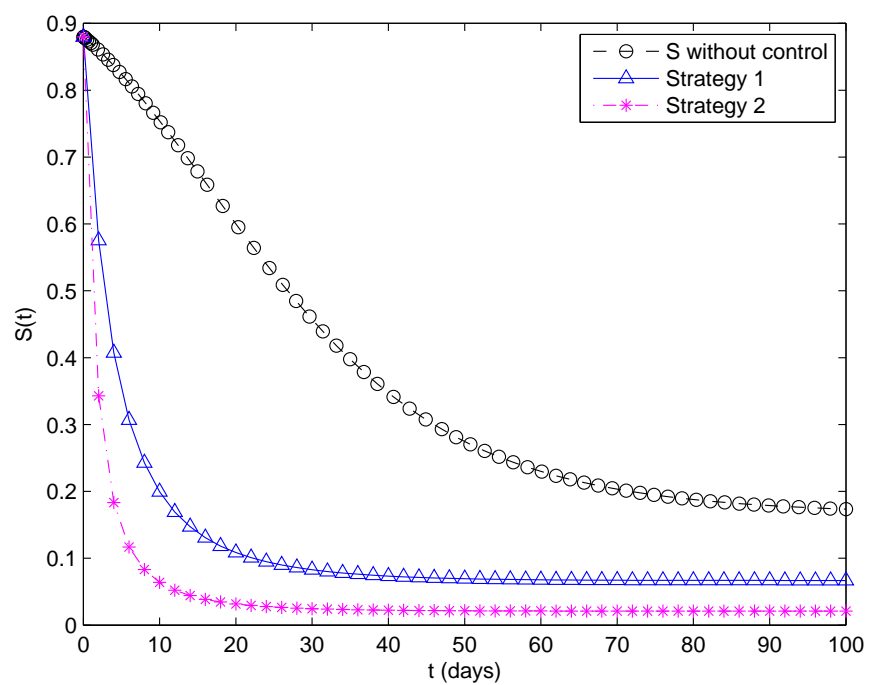

Fig. 2 Comparison between the curves of susceptible individuals $S(t)$ in case of Strategy 1 and Strategy 2 versus without control.

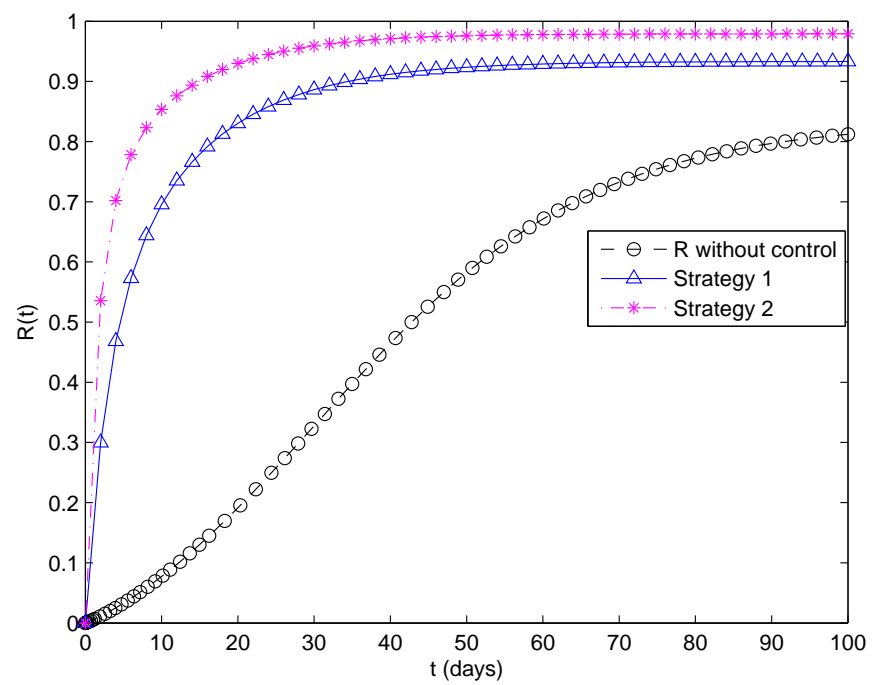

Fig. 3 Comparison between the curves of recovered individuals $R(t)$ in case of Strategy 1 and Strategy 2 versus without control. 


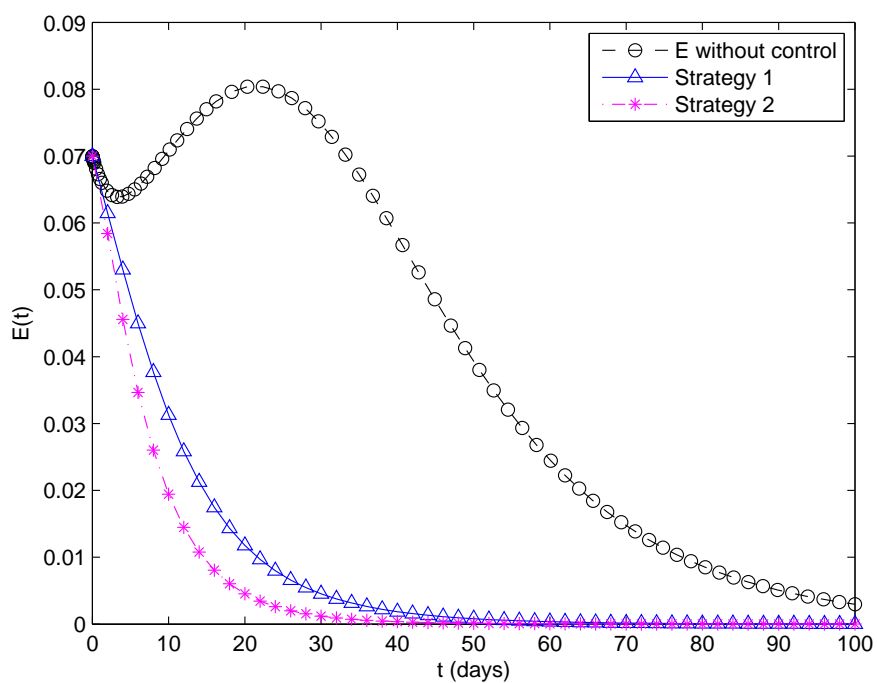

Fig. 4 Comparison between the curves of exposed individuals $E(t)$ in case of Strategy 1 and Strategy 2 versus without control.

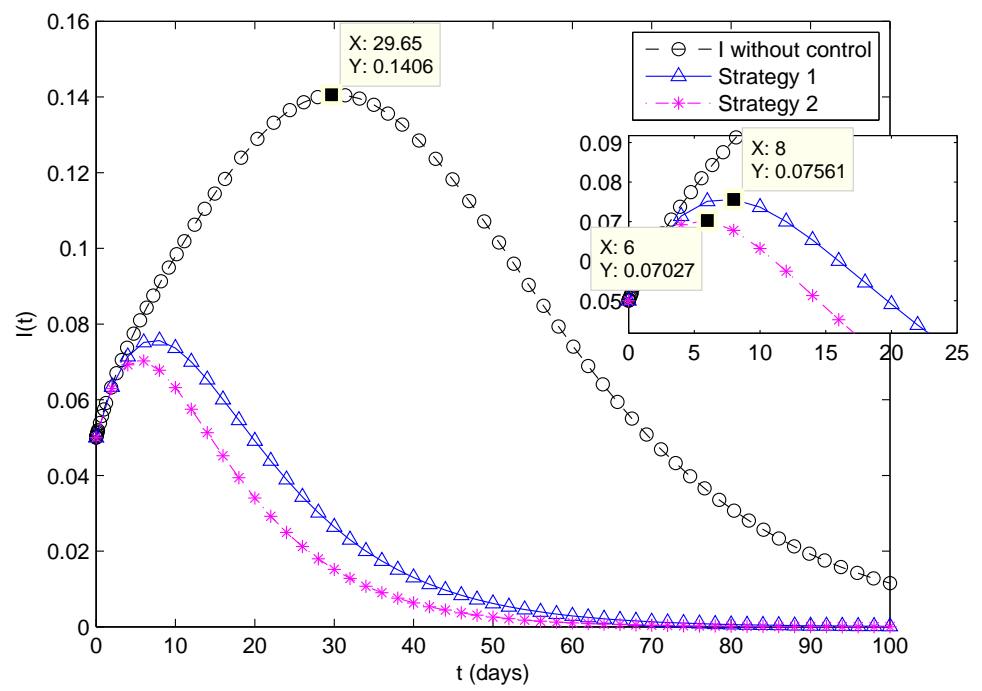

Fig. 5 Comparison between the curves of infected individuals $I(t)$ in case of Strategy 1 and Strategy 2 versus without control. 


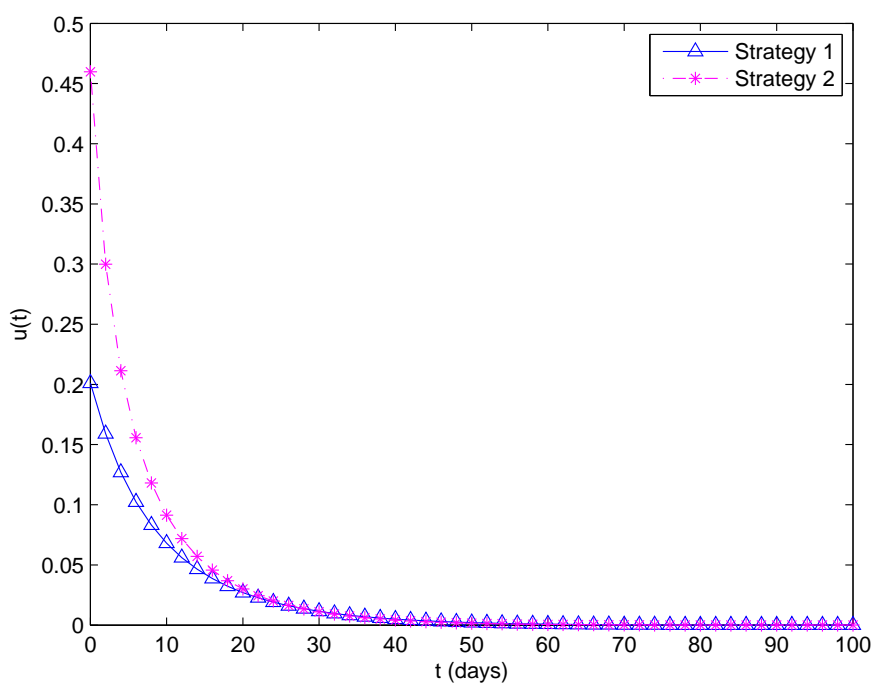

Fig. 6 The optimal control $u$ for Strategy 1 and Strategy 2 .

for the virus. Our idea is to study the effect of educational campaigns with a vaccination treatment in practical Ebola situations. The case of the French nurse cured of Ebola is a proof of the possibility of educational campaigns and treatment [32]. An educational campaign, in case of spread of Ebola, has great importance. In fact, Ebola virus spreads through human-to-human transmission, not only by close and direct physical contact with infected bodily fluids, but also via exposure to objects or contaminated environment. The most infectious fluids are blood, feces, and vomit secretions. However, all body fluids have the capacity to transmit the virus. Here, we intend to control the propagation of the Ebola virus by using two control variables in the SEIR model, as follows:

$$
\left\{\begin{array}{l}
\frac{d S(t)}{d t}=-\beta S(t) I(t)-u_{2}(t) S(t) \\
\frac{d E(t)}{d t}=\beta S(t) I(t)-\gamma E(t) \\
\frac{d I(t)}{d t}=\gamma E(t)-\mu I(t)-u_{1}(t) I(t), \\
\frac{d R(t)}{d t}=\mu I(t)+u_{1}(t) I(t)+u_{2}(t) S(t),
\end{array}\right.
$$

where $u_{1}(t)$ is the fraction of infective that is treated at time $t$, and $u_{2}(t)$ is the fraction of susceptible individuals that is subject to an educational campaign at time $t$. Our goal is to minimize simultaneously the total number of individuals that are infected, the cost of treatment, and the cost of educational campaigns 
to the population. The objective functional is now

$$
J(u)=\int_{0}^{t_{\text {end }}}\left[\kappa I(t)+B_{1} \frac{u_{1}^{2}(t)}{2}+B_{2} \frac{u_{2}^{2}(t)}{2}\right] d t
$$

subject to system (6), where $u=\left(u_{1}, u_{2}\right)$, with $u_{1}$ representing treatment and $u_{2}$ educational campaigns, and $\kappa, B_{1}$ and $B_{2}$ are weight parameters. The Lebesgue measurable control set is defined as

$$
\mathcal{U}_{a d}:=\left\{u=\left(u_{1}, u_{2}\right): u \in L^{1}, 0 \leq u_{1}(t), u_{2}(t) \leq u_{\max }, t \in\left[0, t_{\text {end }}\right]\right\},
$$

where $u_{\max }=0.9$. We choose quadratic terms with respect to the controls in order to describe the nonlinear behaviour of the cost of implementing the educational campaign and treatments. The first term in the objective functional (7), $\kappa I$, stands for the total number of individuals that are infected; the term $B_{1} u_{1}^{2} / 2$ represents the cost of treatment; while the term $B_{2} u_{2}^{2} / 2$ represents the cost associated with the educational campaign. Figure 7 shows the time-dependent curve of susceptible individuals, $S(t)$, which decreases more rapidly in case of the optimal control with Strategy 3. It reaches $1 \%$ at the end of the campaign, instead of $6.6 \%$ at the end of the campaign in case of Strategy 1, and against $17.3 \%$ in the absence of optimal control. Figure 8 shows that the number of recovered individuals of Strategy 3 increases rapidly until $98.9 \%$, instead of $93.3 \%$ in case of Strategy 1, and against $81.2 \%$ without control. Figure 9 shows that the number of exposed individuals of Strategy 3 increases the most rapidly. The period of incubation of the virus is the lest important (30 days) instead of 60 days in case of Strategy 1. In Figure 10 we see the time-dependent curve of infected individuals $I(t)$, which decreases mostly. The other important effect of the Strategy 3, which we can see in the same curve, is the period of infection, which is the less important. The value of the period of infection is 30 days in case of Strategy 3, instead of 80 days in case of Strategy 1, and against 100 days without vaccination. This shows the efficiency of the effect of educational campaigns in controlling Ebola virus with the vaccination control treatment described in Strategy 3. Figure11 gives a representation of the optimal control variables $u_{1}(t)$ and $u_{2}(t)$ of Strategy 3. The minimum of the $J(u)$ functional (7) of Strategy 3 is 0,94 .

\section{Discussion}

In this section we compare between the three strategies of Section 3, and we discuss the obtained results. Figures 12 and 13 represent, respectively, the time-dependent curve of susceptible $S$ and recovered individuals $R$. The curves of $S$ and $R$ of Strategy 3 are slightly greater than the curves of $S$ and $R$ of Strategy 2, which is logical because in the cost functional of Strategy 2 we minimize the infected and the exposed individuals with vaccination, where in the cost functional of Strategy 3 we minimize the infected number with the cost of educational campaigns and vaccination. It is important in the comparison 


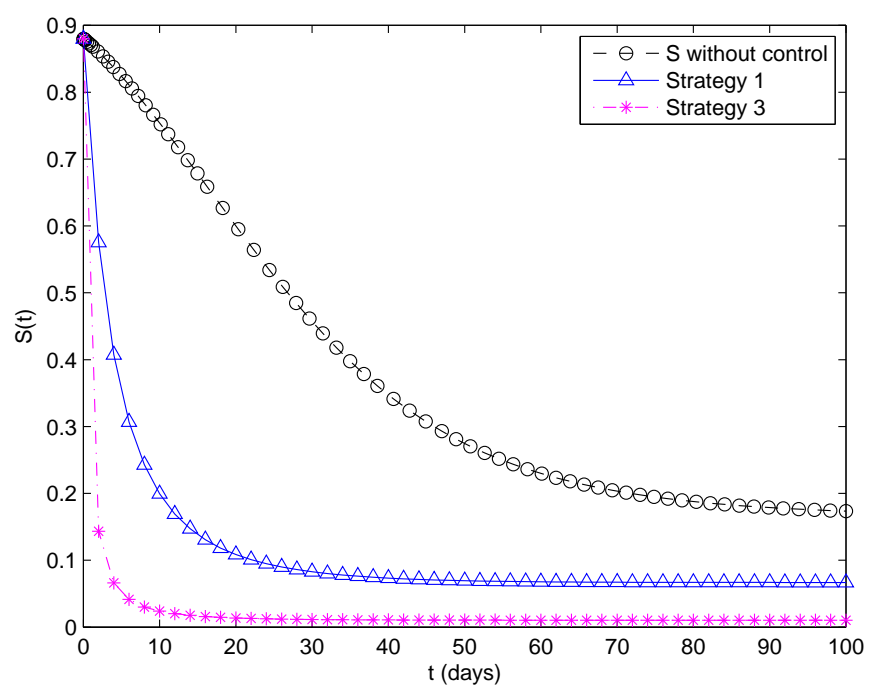

Fig. 7 Comparison between the curves of susceptible individuals $S(t)$ in case of Strategy 1 and Strategy 3 versus without control.

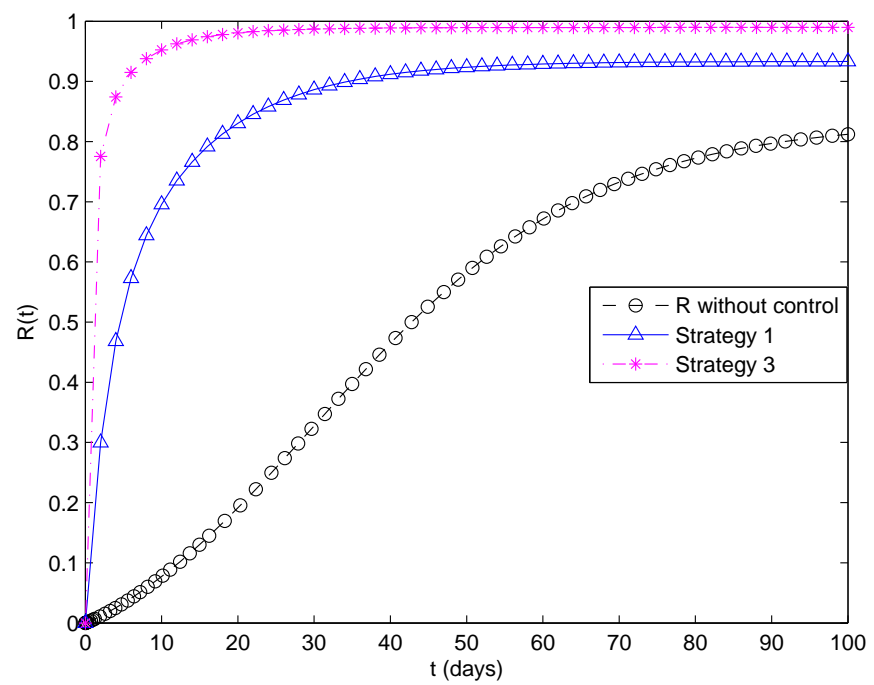

Fig. 8 Comparison between the curves of recovered individuals $R(t)$ in case of Strategy 1 and Strategy 3 versus without control. 


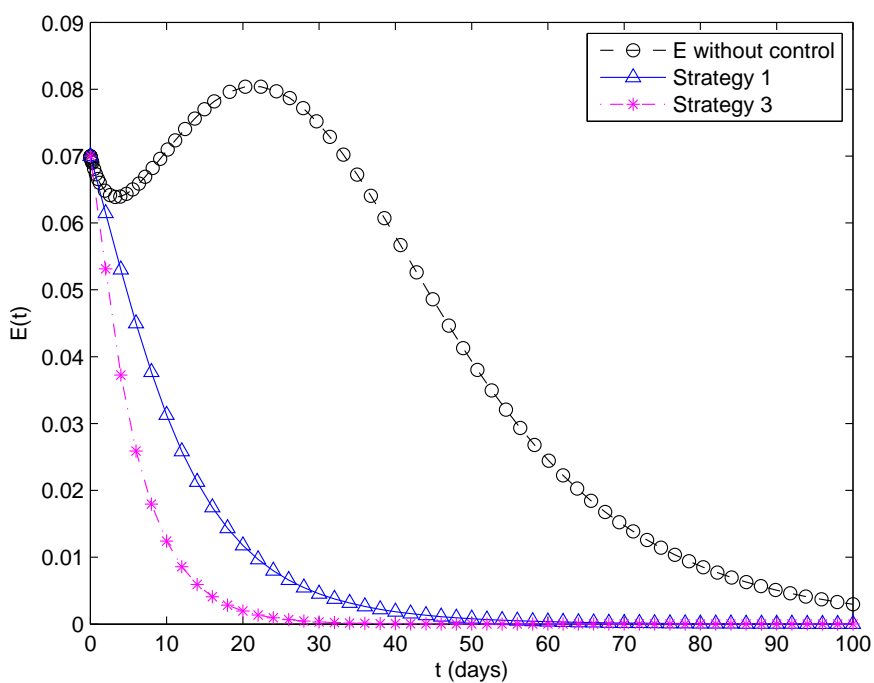

Fig. 9 Comparison between the curves of exposed individuals $E(t)$ in case of Strategy 1 and Strategy 3 versus without control.

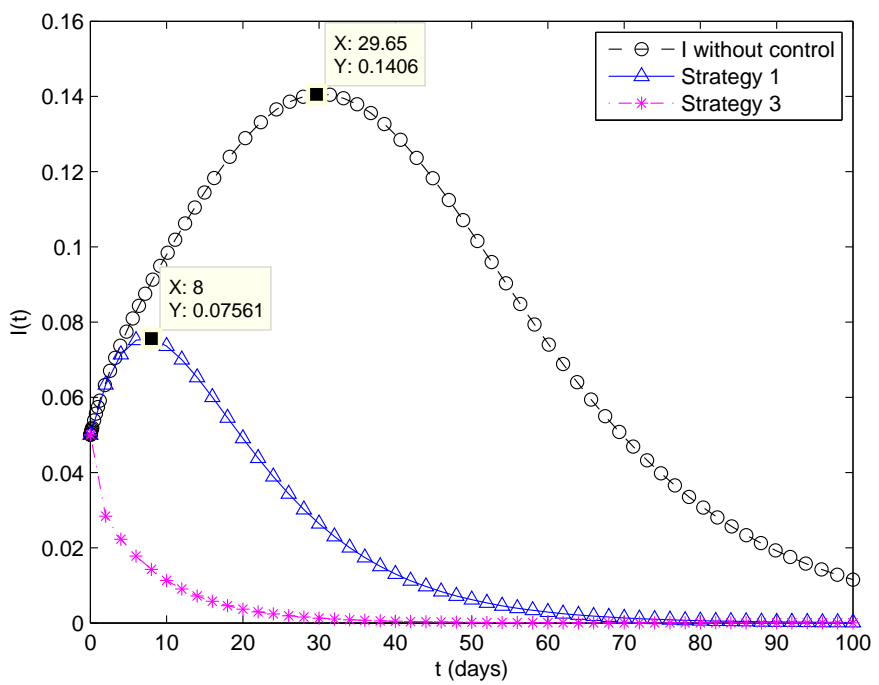

Fig. 10 Comparison between the curves of infected individuals $I(t)$ in case of Strategy 1 and Strategy 3 versus without control. 


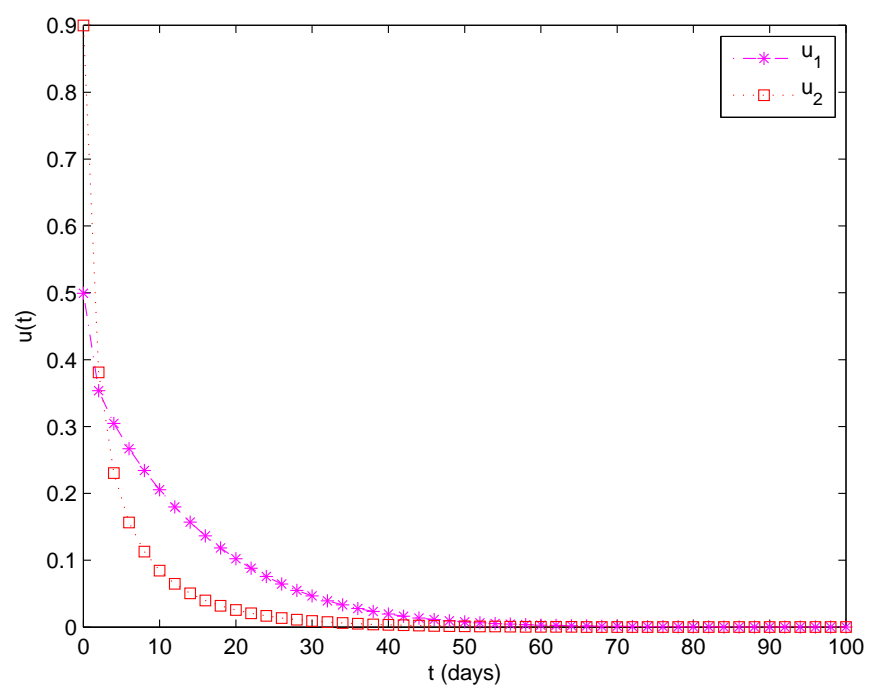

Fig. 11 The optimal control variables $u_{1}$ and $u_{2}$ for Strategy 3 .

between the three curves to note that the number of exposed and infected individuals decrease the most rapidly in case of Strategy 3 (see Figures 14 and 15). Moreover, there is not any peak in case of Strategy 3. The results show the efficiency of an educational campaign in controlling Ebola virus when we couple it with the vaccination treatment control (as given in Strategy 3). We conclude that one can improve vaccination by educational campaigns, which has a great importance in poor countries who do not have the capacity to defend themselves against the virus.

\section{Conclusion}

We studied several optimal control problems for the SEIR model recently discussed by Rachah and Torres in 22, which provides a good description of the 2014 Ebola outbreak in West Africa. Precisely, we introduced a control $u(t)$ representing the vaccination rate at time $t$ (Section 3.1). Then we addressed the problem of reducing not only the number of infected individuals $I(t)$ but also the number of exposed individuals $E(t)$ (Section 3.2). Finally, we investigated the integration of an educational campaign about the virus into the population (Section 3.3). It has been shown that an educational campaign has a great importance with the vaccination treatment, mainly in countries that do not have the capacity to defend themselves against the virus. As future work, we plan to include in our study other factors. For instance, we intend to include in the mathematical model a quarantine procedure. 


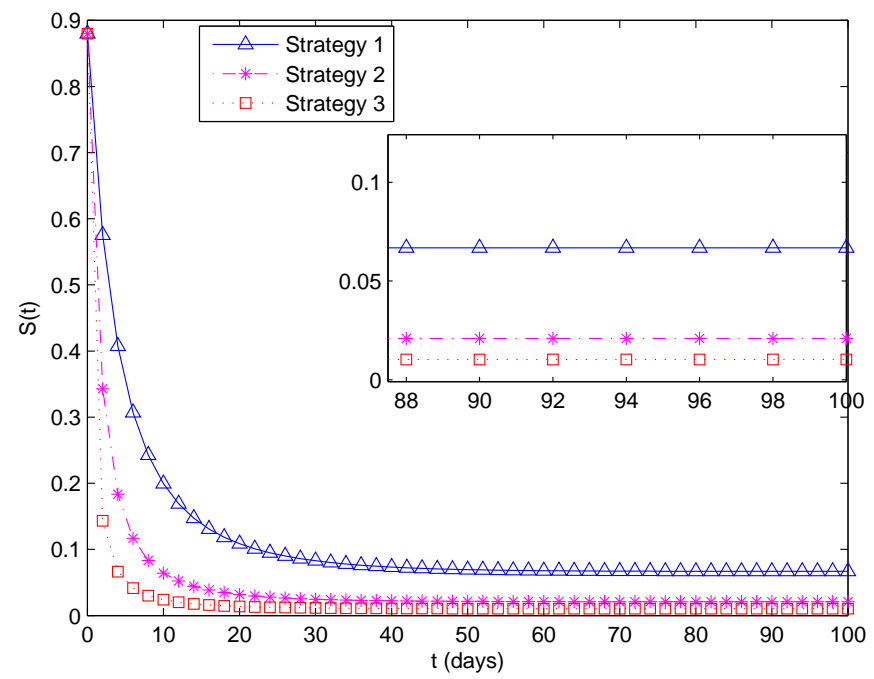

Fig. 12 Comparison between the curves of susceptible individuals $S(t)$ in case of Strategy 1, Strategy 2, and Strategy 3.

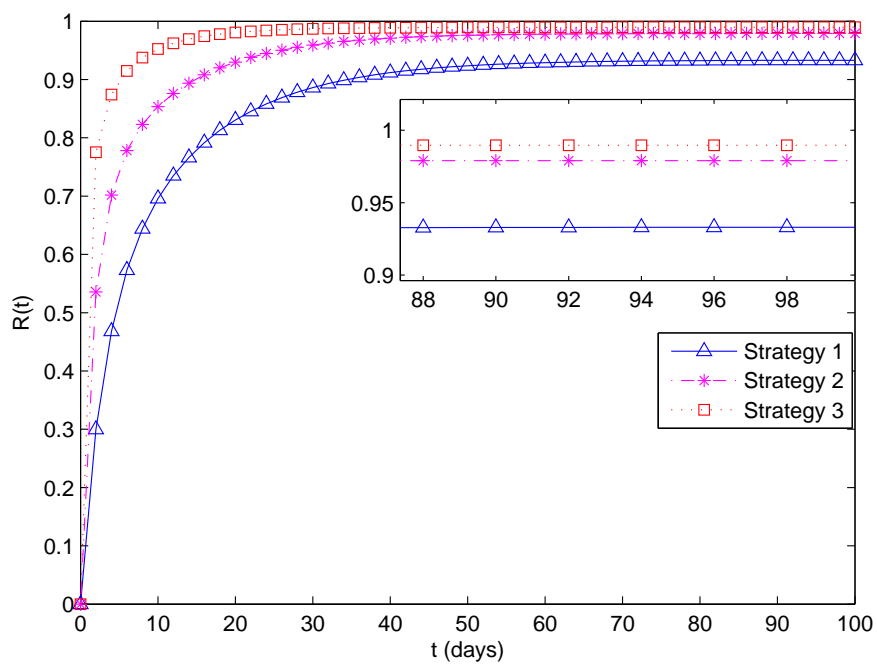

Fig. 13 Comparison between the curves of recovered individuals $R(t)$ in case of Strategy 1, Strategy 2, and Strategy 3. 


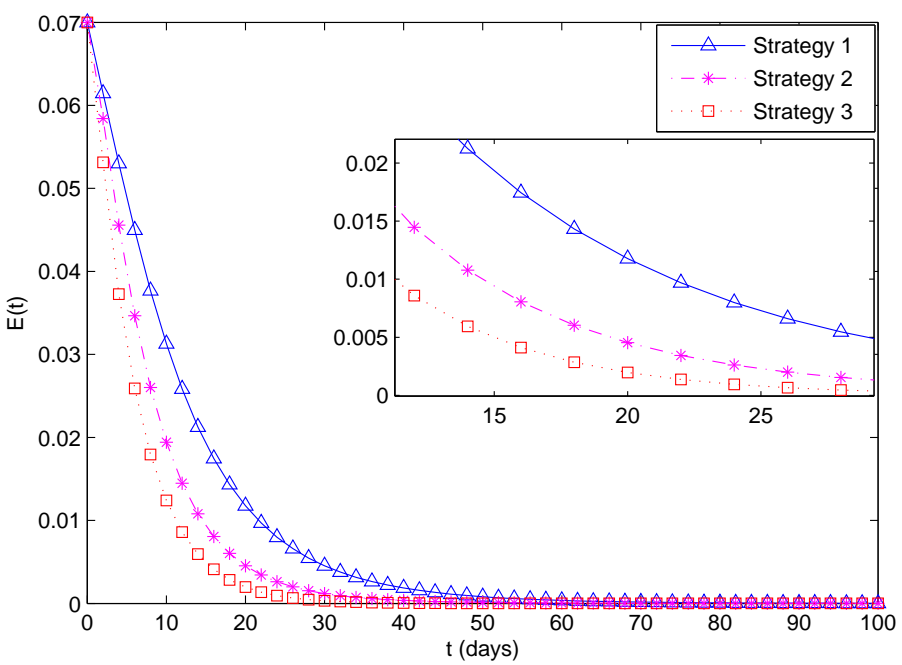

Fig. 14 Comparison between the curves of exposed individuals $E(t)$ in case of Strategy 1, Strategy 2, and Strategy 3.

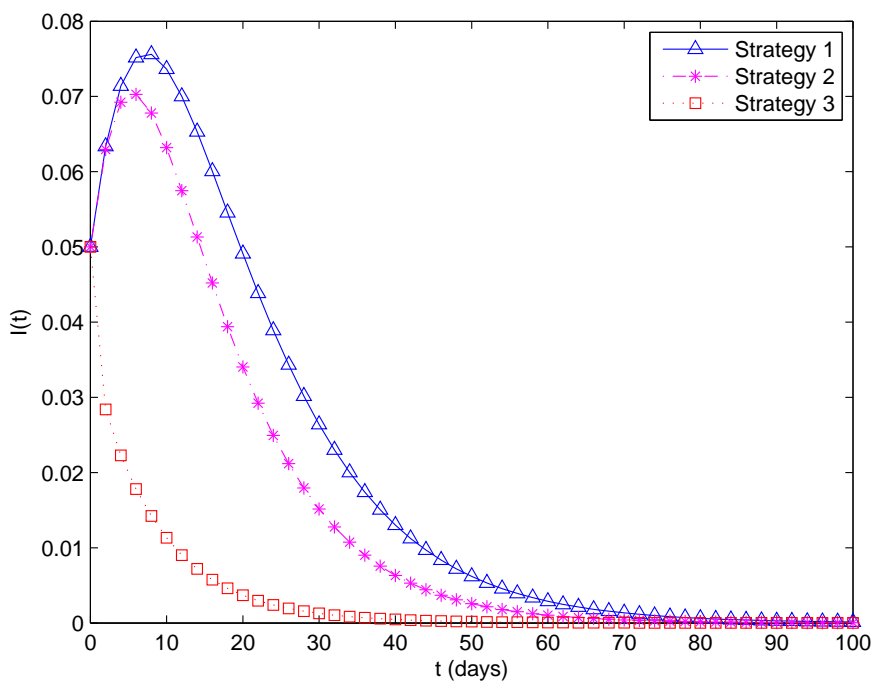

Fig. 15 Comparison between the curves of infected individuals $I(t)$ in case of Strategy 1 , Strategy 2, and Strategy 3. 
Acknowledgements This research was partially supported by the Institute of Mathematics of Toulouse, France (Rachah); and by the Portuguese Foundation for Science and Technology (FCT), within CIDMA project UID/MAT/04106/2013 and OCHERA project PTDC/ EEI-AUT/1450/2012, co-financed by FEDER under POFC-QREN with COMPETE reference FCOMP-01-0124-FEDER-028894 (Torres). The authors would like to thank two referees for valuable comments and helpful suggestions.

\section{References}

1. C. L. Althaus. Estimating the reproduction number of Ebola virus (EBOV) during the 2014 outbreak in West Africa. PLOS Currents Outbreaks, September 2, 2014. http://dx.doi.org/10.1371/currents.outbreaks.91afb5e0f279e7f29e7056095255b288

2. I. Area, H. Batarfi, J. Losada, J. J. Nieto, W. Shammakh, A. Torres. On a fractional order Ebola epidemic model, Adv. Difference Equ. 2015, 2015:278, 12 pp.

3. I. Area, J. Losada, F. Ndaïrou, J. J. Nieto, D. D. Tcheutia. Mathematical modeling of 2014 Ebola outbreak. Math. Methods Appl. Sci., in press. DOI: 10.1002/mma.3794

4. D. Ariens, B. Houska, H. J. Ferreau. ACADO toolkit user's manual. Toolkit for Automatic Control and Dynamic Optimization, 2010. http://www.acadotoolkit.org

5. J. Astacio, D. Briere, M. Guilléon, J. Martinez, F. Rodriguez, N. Valenzuela-Campos. Mathematical models to study the outbreaks of Ebola. Report BU-1365-M, Mathematical and Theoretical Biology Institute (MTBI), Cornell University, 1996.

6. H. T. Banks and C. Castillo-Chavez. Bioterrorism. Frontiers in Applied Mathematics, 28, SIAM, Philadelphia, PA, 2003.

7. M. Barry, F. A. Traoré, F. B. Sako, D. O. Kpamy, E. I. Bah, M. Poncin, S. Keita M. Cisse, A. Touré. Ebola outbreak in Conakry, Guinea: epidemiological, clinical, and outcome features. Médecine et Maladies Infectieuses 44 (2014), no. 11-12, 491-494.

8. C. M. Berry. Vaccine control of avian influenza H5N1 in poultry: need for a positive marker. J. Vaccines Vaccin. 4 (2013), no. 1, Art. ID 1000168, 4 pp.

9. H. G. Bock, K. J. Pitt. A multiple shooting algorithm for direct solution of optimal control problems. Proc. 9th IFAC World Congress, Budapest. Pergamon Press, 243$247,1984$.

10. L. Borio et al. [Working Group on Civilian Biodefense; Corporate Author]. Hemorrhagic fever viruses as biological weapons: medical and public health management. Journal of the American Medical Association 287 (2002), no. 18, 2391-2405.

11. G. Chowell, J. M. Hayman, L. M. A. Bettencourt, C. Castillo-Chavez. Mathematical and statistical estimation approaches in epidemiology, Springer, Dordrecht, 2009.

12. G. Chowell, N. W. Hengartner, C. Castillo-Chavez, P. W. Fenimore, J. M. Hyman. The basic reproductive number of Ebola and the effects of public health measures: the cases of Congo and Uganda. Journal of Theoretical Biology 229 (2004), no. 1, 119-126.

13. S. F. Dowell, R. Mukunu, T. G. Ksiazek, A. S. Khan, P. E. Rollin, C. J. Peters Transmission of Ebola hemorrhagic fever: a study of risk factors in family members, Kikwit, Democratic Republic of the Congo, 1995. Commission de Lutte contre les Epidémies à Kikwit. J. Infect. Dis. 179 (1999), Suppl. 1, S87-S91.

14. S. Gao, Z. Teng, J. J. Nieto, A. Torres. Analysis of an SIR epidemic model with pulse vaccination and distributed time delay. J. Biomed. Biotechnol. 2007 (2007), Art. ID 64870, 10 pp.

15. B. Houska, H. J. Ferreau, M. Diehl. ACADO toolkit - an open-source framework for automatic control and dynamic optimization, Optimal Control Appl. Methods 32 (2011), no. 3, 298-312.

16. E. Jung, S. Iwami, Y. Takeuchi, T.-C. Jo. Optimal control strategy for prevention of avian influenza pandemic. J. Theoret. Biol. 260 (2009), no. 2, 220-229.

17. V. Kaurov. Modeling a pandemic like Ebola with the Wolfram language. Technical Communication \& Strategy, November 4, 2014. http://blog.wolfram.com/2014/11/04/modeling-a-pandemic-like-ebola-with-the-wolfram-language

18. J. Legrand, R. F. Grais, P. Y. Boelle, A. J. Valleron, A. Flahault. Understanding the dynamics of Ebola epidemics. Epidemiol. Infect. 135 (2007), no. 4, 610-621. 
19. J. A. Lewnard, M. L. Ndeffo Mbah, J. A. Alfaro-Murillo, F. L. Altice, L. Bawo, T. G. Nyenswah, A. P. Galvani. Dynamics and control of Ebola virus transmission in Montserrado, Liberia: a mathematical modelling analysis. The Lancet Infectious Diseases 14 (2014), no. 12, 1189-1195.

20. C. J. Peters, J. W. LeDuc. An introduction to Ebola: the virus and the disease. Journal of Infectious Diseases 179 (1999), Suppl. 1, ix-xvi.

21. A. Rachah, D. F. M. Torres. Mathematical modelling, simulation, and optimal control of the 2014 Ebola outbreak in West Africa. Discrete Dyn. Nat. Soc. 2015 (2015), Art. ID 842792, 9 pp. arXiv:1503.07396

22. A. Rachah, D. F. M. Torres. Modelling and numerical simulation of the recent outbreak of Ebola. In: Proceedings of the 2nd International Conference on Numerical and Symbolic Computation: Developments and Applications (SYMCOMP 2015), Universidade do Algarve, Faro, March 26-27, 2015. Edited by APMTAC (Editors: A. Loja, J. I. Barbosa and J. A. Rodrigues), pp. 179-190. ISBN 978-989-96264-7-8.

23. A. Rachah, D. F. M. Torres. Predicting and controlling the Ebola infection. Math. Methods Appl. Sci., in press. DOI: 10.1002/mma.3841 arXiv:1511.06323

24. Report of an International Commission. Ebola haemorrhagic fever in Zaire, 1976. Bull. World Health Organ. 56 (1978), no. 2, 271-293.

25. H. S. Rodrigues, M. T. T. Monteiro, D. F. M. Torres. Dynamics of dengue epidemics when using optimal control. Math. Comput. Modelling 52 (2010), no. 9-10, 1667-1673. arXiv:1006.4392

26. H. S. Rodrigues, M. T. T. Monteiro, D. F. M. Torres. Vaccination models and optimal control strategies to dengue. Math. Biosci. 247 (2014), no. 1, 1-12. arXiv:1310.4387

27. H. S. Rodrigues, M. T. T. Monteiro, D. F. M. Torres. Optimal Control and Numerical Software: An Overview. In: Systems Theory: Perspectives, Applications and Developments (Ed.: F. Miranda), Nova Science Publishers, New York, 2014, 93-110. arXiv:1401.7279

28. P. Rodrigues, C. J. Silva, D. F. M. Torres. Cost-effectiveness analysis of optimal control measures for tuberculosis. Bull. Math. Biol. 76 (2014), no. 10, 2627-2645. arXiv:1409.3496

29. C. J. Silva, D. F. M. Torres. Optimal control for a tuberculosis model with reinfection and post-exposure interventions. Math. Biosci. 244 (2013), no. 2, 154-164. arXiv:1305.2145

30. J. M. Tchuenche, S. A. Khamis, F. B. Agusto, S. C. Mpeshe. Optimal control and sensitivity analysis of an influenza model with treatment and vaccination. Acta Biotheoretica 59 (2011), no. 1, 1-28.

31. Uganda Ministry of Health. An outbreak of Ebola in Uganda. Trop. Med. Int. Health. 7 (2002), no. 12, 1068-1075.

32. A. J. Valleron, D. Schwartz, M. Goldberg, R. Salamon. Collectif Lépidémiologie humaine, Conditions de son développement en France, et rôle des mathématiques. Institut de France Académie des Sciences, Vol. 462, 2006.

33. WHO. Report of an International Study Team. Ebola haemorrhagic fever in Sudan 1976. Bull. World Health Organ. 56 (1978), no. 2, 247-270.

34. WHO. Ebola data and statistics. http://apps.who.int/gho/data/node.ebola-sitrep

35. D. Zeng, H. Chen, C. Castillo-Chavez, W. B. Lober, M. Thurmond. Infectious disease informatics and biosurveillance. Integrated Series in Information Systems, Vol. 27, Springer, New York, 2011. 